\title{
Relationship between senile osteoporosis and cardiovascular and cerebrovascular diseases
}

\author{
XIAOYING HU, SHUCAN MA, CAIXIA YANG, WEIWEI WANG and LIMAN CHEN
}

The First Department of Geriatric Medicine, Harrison International Peace Hospital, Hengshui, Hebei 053000, P.R. China

Received November 20, 2018; Accepted March 18, 2019

DOI: $10.3892 /$ etm.2019.7518

\begin{abstract}
The relationship between senile osteoporosis and cardiovascular hypertension, coronary heart disease and cerebral infarction was investigated. A retrospective study on 428 elderly patients hospitalized in Harrison International Peace Hospital from June 2014 to January 2017 was conducted. There were 207 cases of coronary heart disease, 102 cases of hypertension and 119 cases of cerebral infarction. According to bone density measurement results, the subjects were divided into the osteoporosis group and the non-osteoporosis group. Risk factors for osteoporosis were analyzed, and the incidence of osteoporosis in hypertension, coronary heart disease, and cerebral infarction populations of different severity was analyzed. Hypertension, coronary heart disease and cerebral infarction were the main risk factors for osteoporosis in the elderly. Incidence of osteoporosis in the double-vessel disease group and the three-vessel disease group was significantly higher than that in the single-vessel disease group. Incidence of osteoporosis was significantly higher in the three-vessel disease group than that in the double-vessel disease group $(\mathrm{P}<0.05)$. Incidence of osteoporosis was significantly higher in the moderate hypertension and severe hypertension groups than that in the mild hypertension group. Incidence of osteoporosis was significantly higher in patients with severe hypertension than that in the moderate hypertension group $(\mathrm{P}<0.05)$. Incidence of osteoporosis in patients with moderate cerebral infarction and severe cerebral infarction was significantly higher than that in the mild cerebral infarction group $(\mathrm{P}<0.05)$. Incidence of osteoporosis in patients with severe cerebral infarction was significantly higher than that in the moderate cerebral infarction group $(\mathrm{P}<0.05)$. The results indicated that there is a close correlation between senile osteoporosis and hypertension, coronary heart disease and cerebral infarction. Osteoporosis can be used as a predictor of early screening for
\end{abstract}

Correspondence to: Dr Caixia Yang, The First Department of Geriatric Medicine, Harrison International Peace Hospital, 180 Renmin East Road, Taocheng, Hengshui, Hebei 053000, P.R. China

E-mail: wy47g3@163.com

Key words: elderly population, osteoporosis, hypertension, coronary heart disease, cerebral infarction hypertension, coronary heart disease and cerebral infarction in the elderly population.

\section{Introduction}

Osteoporosis is an orthopaedic disease characterized by reduced bone mass and microstructural degradation of bone. Osteoporosis can be divided into primary and secondary types, of which primary osteoporosis is the main type in clinical practice $(1,2)$. Primary osteoporosis mainly affects the elderly population. With the growth of aging population in China, the incidence of osteoporosis also shows an increasing trend. The risk of cardiovascular disease is increased with aging. Common cardiovascular diseases in China include hypertension, coronary heart disease, and cerebral infarction. Previous studies have revealed that many elderly patients with osteoporosis are associated with cardiovascular diseases such as hypertension, coronary heart disease, and cerebral infarction. Osteoporosis and various cardiovascular diseases have a common pathophysiological basis $(3,4)$. However, studies on the correlation between senile osteoporosis and cardiovascular and cerebrovascular diseases, especially the correlation between osteoporosis and the severity of cardiovascular and cerebrovascular diseases are rare. In this study, patients with hypertension, coronary heart disease and cerebral infarction were used as the research subjects to explore the correlation between senile osteoporosis and cardiovascular disease. This study provides a theoretical basis for screening high-risk groups of cardiovascular and cerebrovascular diseases based on the diagnosis of early osteoporosis.

\section{Patients and methods}

Research subjects. A retrospective study on 600 elderly patients hospitalized in Harrison International Peace Hospital (Hengshui, China) from June 2014 to January 2017 was conducted. There were 207 cases of coronary heart disease, 102 cases of hypertension and 119 cases of cerebral infarction. Two hundreds and seven patients with coronary heart disease including 120 males and 87 females, aged 60-81 years, with a mean age of $68.85 \pm 6.52$ years. A hundred and two patients with hypertension including 62 males and 40 females, aged 60-80 years, with a mean age of $67.73 \pm 6.58$ years. A hundred and nineteen cases of cerebral infarction including 
60 males and 59 females, aged $61-79$ years, with a mean age of $67.48 \pm 6.38$ years. Coronary heart disease was diagnosed according to the guidelines for the diagnosis and treatment of chronic stable angina pectoris established by the Chinese Medical Association Cardiovascular Branch. Cerebral infarction was diagnosed according to the 2010 guidelines for the diagnosis and treatment of acute ischemic stroke in China.

Inclusion criteria. Inclusion criteria were: i) patients with complete medical records; ii) patients older than 60 years old; iii) patients with no chronic wasting disease and malignant tumors, and iv) patients with no autoimmune disease and infectious diseases.

Exclusion criteria. Exclusion criteria were: i) patients with thyroid or parathyroid disease; ii) patients with renal tubular and glomerular diseases; iii) patients with diabetes or chronic liver disease; iv) patients with bone metastases and osteoma; v) patients with a history of abdominal surgery; vi) patients who were combined with other serious physical diseases and other liver, kidney, and endocrine diseases that affect bone metabolism; vii) patients who were taking hormones or other drugs that affect bone metabolism; viii) patients with a history of mental illness, and ix) patients with incomplete medical record.

The study was approved by the Ethics Committee of Harrison International Peace Hospital. Signed informed consents were obtained from the patients or guardians.

Severity of coronary artery disease. Left and right coronary arteries of patients with coronary heart disease were imaged by contrast instrument. Severity of coronary artery disease was evaluated according to the results of coronary angiography. According to coronary artery lesions, patients were divided into the single-vessel disease group, the double-vessel disease group and the three-vessel disease group (5).

Severity of hypertension. Blood pressure of hypertension patients was measured according to standard blood pressure measurement method, and the severity of hypertension was evaluated according to the measured diastolic blood pressure and systolic blood pressure. Mild hypertension: diastolic blood pressure is $90-99 \mathrm{mmHg}$ and systolic blood pressure is $140-159 \mathrm{mmHg}$. Moderate hypertension: diastolic blood pressure is $100-109 \mathrm{mmHg}$ and systolic blood pressure is 160-179 mmHg. Severe hypertension: diastolic blood pressure $\geq 110 \mathrm{mmHg}$ and systolic blood pressure $\geq 180 \mathrm{mmHg}$ (6).

Severity of cerebral infarction. The US National Institutes of Health Stroke Scale was used to assess the severity of cerebral infarction patients. Mild cerebral infarction: 2-4 points, moderate cerebral infarction: 5-20 points, severe cerebral infarction: $>21$ points (7).

Bone density measurement. Lumbar spine and left hip bone mineral density of all subjects were measured using a dual-energy X-ray density meter, and the results were compared with the peak bone mass $(\mathrm{T})$ of healthy individuals of the same sex. According to the Chinese Medical Association's guidelines for clinical diagnosis and treatment of osteoporosis, the criterion for the determination of bone mass is $\mathrm{T}$ score. $\mathrm{T}<-1.0$ indicates normal bone mass. Bone mass is reduced when $\mathrm{T}>-1.0-<-2.5$. Osteoporosis was diagnosed when $\mathrm{T} \geq-2.5$. According to the bone mineral density measurement results of the study subjects and the diagnostic criteria for osteoporosis, patients with hypertension, coronary heart disease and cerebral infarction were divided into the osteoporosis and non-osteoporosis groups (8).

Statistical analysis. Statistical analyses were performed using SPSS 19.0 statistical software (IBM Corp., Armonk, NY, USA). Count data were expressed as $\%$ and the differences between groups were compared by $\chi^{2}$ test. Factors affecting osteoporosis were analyzed by Logistic regression analysis, and the test level was $\alpha=0.05$. Differences were statistically significant at $\mathrm{P}<0.05$.

\section{Results}

Logistic regression analysis of senile osteoporosis and cardiovascular and cerebrovascular diseases. With osteoporosis as a dependent variable, BMI, blood glucose, triglyceride, cholesterol, high-density lipoprotein, low-density lipoprotein, serum calcium, serum enzymes, hypertension, coronary heart disease, cerebral infarction and other factors were introduced into the two-class logistic model. Results showed that hypertension, coronary heart disease and cerebral infarction are the main risk factors for osteoporosis in the elderly (Table I).

Comparison of incidence of osteoporosis in patients with coronary heart disease of different severity. Incidence of osteoporosis in patients with different severity of coronary heart disease was compared. Results showed that the incidence of osteoporosis in the double-vessel disease and three-vessel disease groups was significantly higher than that in the single-vessel disease group $(\mathrm{P}<0.05)$. Incidence of osteoporosis in the three-vessel disease group was significantly higher than that in the double-vessel disease group $(\mathrm{P}<0.05)$ (Table II).

Comparison of incidence of osteoporosis in patients with hypertension of different severity. Incidence of osteoporosis in patients with hypertension of different severity was compared. Results showed that incidence of osteoporosis was significantly higher in the moderate hypertension and severe hypertension groups than that in the mild hypertension group $(\mathrm{P}<0.05)$. Incidence of osteoporosis was significantly higher in patients with severe hypertension than that in the moderate hypertension group $(\mathrm{P}<0.05)$ (Table III).

Comparison of incidence of osteoporosis in patients with different severity of cerebral infarction. Incidence of osteoporosis in patients with different severity of cerebral infarction was compared. Results showed that incidence of osteoporosis in patients with moderate cerebral infarction and severe cerebral infarction was significantly higher than that in the mild cerebral infarction group $(\mathrm{P}<0.05)$. Incidence of osteoporosis in patients with severe cerebral infarction was significantly higher than that in patients with moderate cerebral infarction $(\mathrm{P}<0.05)$ (Table IV). 
Table I. Logistic regression analysis of senile osteoporosis and cardiovascular and cerebrovascular diseases.

\begin{tabular}{|c|c|c|c|c|c|c|}
\hline Factors & $\beta$ & $\mathrm{SE}$ & Wald & P-value & OR & $95 \% \mathrm{CI}$ \\
\hline BMI index & 1.277 & 0.670 & 2.811 & 0.091 & 3.090 & $0.827-11.543$ \\
\hline Blood glucose & 0.891 & 0.342 & 2.984 & 0.099 & 3.225 & $0.873-10.632$ \\
\hline Triglyceride & 1.046 & 0.568 & 2.764 & 0.064 & 2.536 & $0.672-9.621$ \\
\hline Cholesterol & 1.116 & 0.579 & 3.682 & 0.058 & 2.791 & $0.566-7.287$ \\
\hline High-density lipoprotein & 1.268 & 0.683 & 2.578 & 0.068 & 2.686 & $0.673-9.632$ \\
\hline Low-density lipoprotein & 1.673 & 0.883 & 2.637 & 0.082 & 3.872 & $0.737-6.782$ \\
\hline Serum calcium & 1.767 & 0.577 & 2.687 & 0.067 & 2.683 & $0.472-7.827$ \\
\hline Serum enzyme & 1.350 & 0.737 & 2.673 & 0.073 & 3.682 & $0.682-8.732$ \\
\hline Hypertension & 1.872 & 0.777 & 6.838 & 0.021 & 5.373 & $2.683-14.682$ \\
\hline Coronary heart disease & 2.111 & 0.578 & 8.035 & 0.045 & 8.683 & $2.016-34.626$ \\
\hline Brain infarction & 1.597 & 0.745 & 4.037 & 0.044 & 4.286 & $1.376-20.863$ \\
\hline
\end{tabular}

Table II. Comparison of incidence of osteoporosis in patients with coronary heart disease of different severity.

\begin{tabular}{lcc}
\hline Groups & Cases & Osteoporosis (cases \%) \\
\hline Single-vessel disease & 67 & $15 / 22.39$ \\
Double-vessel disease & 71 & $26 / 36.62^{\mathrm{a}}$ \\
Three-vessel disease & 69 & $34 / 49.28^{\mathrm{a}, \mathrm{b}}$ \\
$\chi^{2}$ & & 10.643 \\
P-value & & 0.005 \\
\hline
\end{tabular}

${ }^{\mathrm{a}} \mathrm{P}<0.05$, compared with the single-vessel disease group; ${ }^{\mathrm{b}} \mathrm{P}<0.05$, compared with the double-vessel disease group.

Table III. Comparison of incidence of osteoporosis in patients with hypertension of different severity.

\begin{tabular}{lcc}
\hline Groups & Cases & Osteoporosis (cases \%) \\
\hline Mild hypertension & 31 & $8 / 25.81$ \\
Moderate hypertension & 40 & $14 / 35.00^{\mathrm{a}}$ \\
Severe hypertension & 31 & $15 / 48.39^{\mathrm{a}, \mathrm{b}}$ \\
$\chi^{2}$ & & 7.257 \\
P-value & & 0.027 \\
\hline
\end{tabular}

${ }^{\mathrm{a}} \mathrm{P}<0.05$, compared with the mild hypertension group; ${ }^{\mathrm{b}} \mathrm{P}<0.05$, compared with the moderate hypertension group.

\section{Discussion}

Aging causes a series of changes in the human body. Organ function reduction during aging leads to cardiovascular and vascular diseases and osteoporosis, which are common diseases in the elderly population. Epidemiological studies have shown a close correlation between cardiovascular and cerebrovascular diseases and osteoporosis $(9,10)$. Incidence of cardiovascular and cerebrovascular diseases such as hypertension, coronary heart disease and cerebral infarction in the
Table IV. Comparison of incidence of osteoporosis in patients with different severity of cerebral infarction.

\begin{tabular}{lcc}
\hline Groups & Cases & Osteoporosis (cases \%) \\
\hline Mild cerebral infarction & 45 & $9 / 20.00$ \\
Moderate cerebral infarction & 33 & $12 / 36.36^{\mathrm{a}}$ \\
Severe cerebral infarction & 41 & $20 / 48.78^{\mathrm{a}, \mathrm{b}}$ \\
$\chi^{2}$ & & 7.943 \\
P-value & & 0.019 \\
\hline
\end{tabular}

${ }^{\mathrm{a}} \mathrm{P}<0.05$, compared with the mild cerebral infarction group; ${ }^{\mathrm{b}} \mathrm{P}<0.05$, compared with the moderate cerebral infarction group.

elderly osteoporosis group is higher than that of the elderly population with normal bone density. In addition, studies have shown that the use of low bone density as a risk factor for cardiovascular and cerebrovascular events and death may predict the occurrence of cardiovascular and cerebrovascular diseases and the progress of the disease and is more accurate than traditional risk factors such as smoking and hyperlipidemia (11). Logistic regression analysis in this study showed that hypertension, coronary heart disease, and cerebral infarction were the main risk factors for osteoporosis in the elderly. Our findings are consistent with previous studies.

Hypertension is caused by a variety of factors such as increased peripheral vascular resistance and increased cardiac output. Renin-angiotensin-aldosterone system plays an important role in the regulation of blood pressure in the human body. Some scholars believe that renin-angiotensin-aldosterone system plays an important role not only in the regulation of blood pressure, but also in bone tissue. Moreover, renin-angiotensin-aldosterone system is also present in bone tissue to regulate the production and absorption of bone cells, and angiotensin I and angiotensin II can also regulate the production and absorption of osteoclasts. Angiotensin II can regulate the proliferation of vascular endothelial cells and the formation of osteoblasts during bone remodeling (12). Animal experiments have shown that the level of angiotensin II in 
rats with essential hypertension increases, while the level of BMD in the bone decreases, the blood vessels have spasms and harden, apoptosis of bone cells increases, the number of osteoblasts decreases, the number of osteoclasts increases, bone mass decreases, and characteristics of osteoporosis can be observed (13). Coronary heart disease is a heart disease characterized by myocardial ischemia, hypoxia or necrosis caused by coronary angiogenic atherosclerotic lesions, which lead to stenosis or obstruction of blood vessels. Osteoporosis patients have decreased conversion rate of osteoclasts to osteoblasts, which in turn lead to increased osteolysis and increased calcium ion concentration in blood circulation. High levels of calcium ions in the blood circulation deposit on the intima of the inner wall of the arterial vessel. Over time, atherosclerosis and calcification of the vessel wall including the coronary artery may occur, eventually leading to coronary heart disease (14). Cerebral infarction is caused by a variety of causes of blood supply disorders in the local brain tissue. Cerebral infarction leads to local tissue ischemia and hypoxia, brain cell necrosis, and impaired neurological function. Increased osteolysis in patients with osteoporosis leads to increased calcium levels in the blood circulation. High levels of calcium ions deposit on the intima of the inner wall of the cerebral blood vessels and cause cerebral vascular atherosclerosis and calcification, eventually leading to reduced blood supply to the brain (15). In this study, it was revealed that with the increase in the severity of hypertension, coronary heart disease and cerebral infarction, incidence of osteoporosis was significantly increased. These data suggest that there is a close correlation between hypertension, coronary heart disease and cerebral infarction and osteoporosis, which can be used to predict the occurrence and development of cardiovascular and cerebrovascular diseases.

In summary, there is a close correlation between senile osteoporosis and hypertension, coronary heart disease and cerebral infarction. Osteoporosis can be used as a predictor of early screening for hypertension, coronary heart disease and cerebral infarction in the elderly population.

\section{Acknowledgements}

Not applicable.

\section{Funding}

No funding was received.

\section{Availability of data and materials}

The datasets used and/or analyzed during the present study are available from the corresponding author on reasonable request.

\section{Authors' contributions}

XH drafted the manuscript. XH and SM recorded and analyzed the severity of hypertension. WW and LC were responsible for the severity of cerebral infarction analysis. SM and CY assisted with bone density measurement. The final version was read and approved by all the authors.

\section{Ethics approval and consent to participate}

The study was approved by the Ethics Committee of Harrison International Peace Hospital (Hengshui, China). Signed informed consents were obtained from the patients or guardians.

\section{Patient consent for publication}

Not applicable.

\section{Competing interests}

The authors declare that they have no competing interests.

\section{References}

1. Ilić K, Obradović N and Vujasinović-Stupar N: The relationship among hypertension, antihypertensive medications, and osteoporosis: A narrative review. Calcif Tissue Int 92: 217-227, 2013.

2. Kuipers AL, Miljkovic I, Carr JJ, Terry JG, Nestlerode CS, Ge Y, Bunker CH, Patrick AL and Zmuda JM: Association of circulating sclerostin with vascular calcification in Afro-Caribbean men. Atherosclerosis 239: 218-223, 2015.

3. Drechsler C, Evenepoel P, Vervloet MG, Wanner C, Ketteler M, Marx N, Floege J, Dekker FW and Brandenburg VM; NECOSAD Study Group: High levels of circulating sclerostin are associated with better cardiovascular survival in incident dialysis patients: Results from the NECOSAD study. Nephrol Dial Transplant 30: 288-293, 2015

4. Drake MT, Clarke BL, Oursler MJ, Khosla S and Cathepsin K: Cathepsin K inhibitors for osteoporosis: Biology, potential clinical utility, and lessons learned. Endocr Rev 38: 325-350, 2017.

5. Chen SJ, Lin CS, Lin CL and Kao CH: Osteoporosis is associated with high risk for coronary heart disease: A population-based cohort study. Medicine (Baltimore) 94: e1146, 2015.

6. Wolley MJ, Pimenta E, Calhoun D, Gordon RD, Cowley D and Stowasser M: Treatment of primary aldosteronism is associated with a reduction in the severity of obstructive sleep apnoea. J Hum Hypertens 31: 561-567, 2017.

7. Fu HJ, Zhao LB, Xue JJ, Wu ZX, Huang YP, Liu W and Gao Z: Elevated serum homocysteine (Hcy) levels may contribute to the pathogenesis of cerebral infarction. J Mol Neurosci 56: 553-561, 2015.

8. Lee SN, Cho JY, Eun YM, Song SW and Moon KW: Associations between osteoporosis and coronary artery disease in postmenopausal women. Climacteric 19: 458-462, 2016.

9. Lash RW, Nicholson JM, Velez L, Van Harrison R and McCort J: Diagnosis and management of osteoporosis. Prim Care 36: 181-198, 2009.

10. Gao M, Tang H, Zheng X, Zhou F and Lu W: Association analysis of GWAS and candidate gene loci in a Chinese population with coronary heart disease. Int J Clin Exp Med 8: 7497-7506, 2015.

11. Kuru P, Akyüz G, Cerşit HP, Çelenlioğlu AE, Cumhur A, Biricik Ş, Kozan S, Gökșen A, Özḑemir M and Lüleci E: Fracture history in osteoporosis: Risk factors and its effect on quality of life. Balkan Med J 31: 295-301, 2014.

12. Shimizu H, Nakagami H, Osako MK, Hanayama R, Kunugiza Y, Kizawa T, Tomita T, Yoshikawa H, Ogihara T and Morishita R: Angiotensin II accelerates osteoporosis by activating osteoclasts. FASEB J 22: 2465-2475, 2008.

13. Pennisi P, Russo E, Gaudio A, Veca R, D'Amico F, Mangiafico RA, Laspina M, Tringali G, Signorelli SS and Fiore CE: The association between carotid or femoral atherosclerosis and low bone mass in postmenopausal women referred for osteoporosis screening. Does osteoprotegerin play a role? Maturitas 67: 358-362, 2010.

14. Ye XL and Lu CF: Association of polymorphisms in the leptin and leptin receptor genes with inflammatory mediators in patients with osteoporosis. Endocrine 44: 481-488, 2013.

15. Zhang J, Xu R, Li Z and Zha W: Cerebral infarction due to malposition of cervical pedicle screw: A case report. Medicine (Baltimore) 97: e9937, 2018.

This work is licensed under a Creative Commons Attribution-NonCommercial-NoDerivatives 4.0 International (CC BY-NC-ND 4.0) License. 